“C 2011 IEEE. Personal use of this material is permitted. Permission from IEEE must be obtained for all other uses, in any current or future media, including reprinting/republishing this material for advertising or promotional purposes, creating new collective works, for resale or redistribution to servers or lists, or reuse of any copyrighted component of this work in other works." 


\section{Semi-Autonomous Competency Assessment of Powered Mobility Device Users}

\author{
Jaime Valls Miro \\ Faculty of Engineering and IT \\ University of Technology Sydney (UTS) \\ Sydney NSW 2007, Australia \\ jaime.vallsmiro@uts.edu.au
}

\author{
Ross Black \\ Occupational Therapy Department \\ Prince of Wales Hospital \\ Sydney NSW 2031 \\ ross.black@sesiahs.health.nsw.gov.au freek.debruijn,gamini.dissanayake@uts.edu.au
}

\begin{abstract}
This paper describes a stand-alone sensor package and algorithms for aiding the assessment by an occupational therapist whether a person has the capacity to safely and effectively operate a powered mobility device such as a walking aid or a wheelchair. The sensor package employed consists of a laser range finder, an RGB camera and an inertial measurement unit that can be attached to any mobility device with minimal modifications. Algorithms for capturing the data received by the sensor package and for generating the map of the environment as well as the trajectory of the mobility device have been developed. Such information presents occupational therapists with the capability to provide a quantitative assessment of whether patients are ready to be safely deployed with mobile aids for their daily activities. Preliminary evaluation of the sensor package and associated algorithms based on experiments, conducted at the premises of the Prince of Wales Hospital in Sydney, are presented.
\end{abstract}

\section{INTRODUCTION}

Research demonstrates that use of appropriate Assistive Technology (AT) is associated with increased independence and reduced need for ongoing care and support [1]. Programs for the service provision of AT maximise the independence of people in our community and are as important as acute and rehabilitation treatment programs [2]. There is an increasing need for assistive technology as the age and number of people with disabilities increases. Powered mobility devices (PMDs) such as electric wheelchairs and scooters are proving to be useful pieces of assistive technology. Adults aged over 50 years are the most prevalent wheelchairs users [3] and it is estimated that PMD use is 3.5 times more frequent after the age of 65 years [4].

Matching the technology of a PMD to user's needs is a long and complex process. The devices are heavy and fast-moving and can be used both in- and outdoors in the presence of static (architectural barriers) and dynamic (pedestrian and vehicular traffic) hazards. In a typical PMD assessment a therapist will observe the client using the device in the environments in which it is to be used. There are a number of areas to consider both before and during the assessment. These variables include:

- user considerations such as experience, motor, sensory, vestibular, cognitive and visual skills

- the device components, the access method, and the seating system [5], and
- the terrain in which the device is to be used.

The device components include the different kinds of devices available and the characteristics, features and idiosyncrasies of each model, number of items that need to be set up on the device (e.g. the seat tilt and backrest, the position of the footplates, arm rest adjustment, seat height), different kinds of "drive configuration" (rear-, mid- and front-wheel drive systems), different kinds of wheelchair controllers and how they are positioned and programmed (i.e. velocity and acceleration during linear and angular motion, and caster and suspension adjustments [6]).

There are multiple aspects to the use and acceptance of AT by users and a number of variables that can be assessed. Generic tools used to assess AT are often subjective (Psychosocial Impact of Assistive Devices (PIADS), the Occupational Therapy Functional Assessment Compilation Tool (OTFACT) Assistive Technology Outcome Measure (ATOM) and The Wheelchair Outcome Measure (WhOM)) and not necessarily specific to the device being assessed [7]. Some wheelchair user-specific questionnaires have been developed to assess self-perceived wheelchair skills e.g. the Wheelchair Skills Test [8] and function related to wheelchair/scooter use e.g. the Functional Evaluation in a Wheelchair [9], Power-Mobility Indoor Driving Assessment (PIDA) [10] and the Power-Mobility Community Driving Assessment (PCDA) [11]. Most organizations and service providers develop their own checklist of parameters to be assessed e.g. Department of Veterans' Affairs "EWC_Scooter Guidelines" 2010 or the "Occupational Therapy Power Wheelchair Assessment". Generally, the models of practice and assessments used for selection of assistive technology are poorly developed and concerns have been raised about the available wheelchair assessments, clearly indicating the need for further research. An extensive review of the literature from 2003-2007 concluded that there is lack of evidencebased procedures for the selection of assistive technology [12]. A "paucity of studies with higher levels of evidence-based practice" was concluded in relation to matching of mobility assistive technology in people with multiple sclerosis [13]. Large inconsistencies have been reported between currently available manual wheelchair tests [14]. Also, commonly used standardized PMD assessments are not intended to determine 


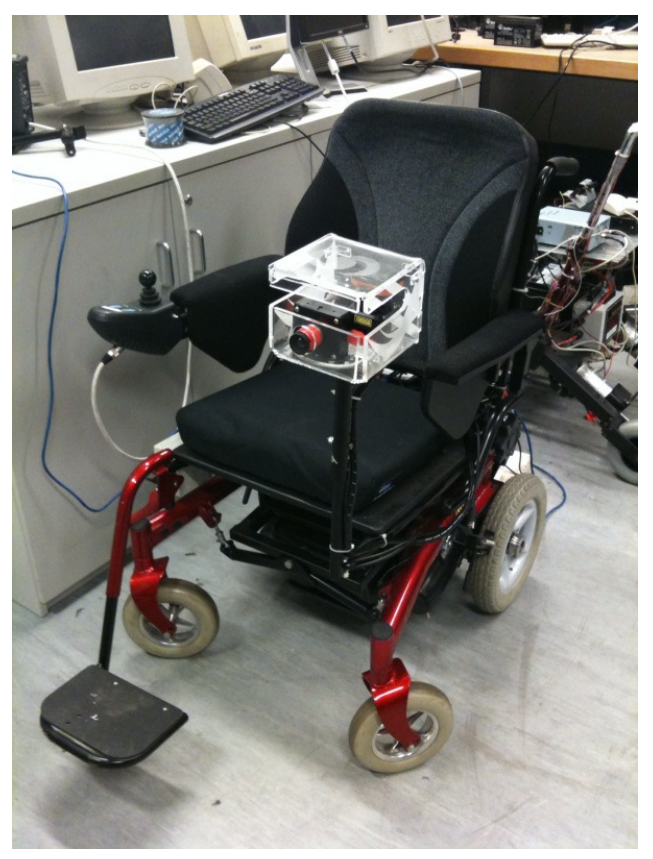

Fig. 1. Sensor package mounted on UTS instrumented wheelchair

whether or not a person will be a safe driver and don't assist therapists in determining when risk becomes untenable [15]. In addition, funding bodies in countries such as Australia are increasingly requiring more detailed and specific data about the intended uses and suitability of expensive pieces of equipment e.g. electric wheelchairs and scooters, before they will support therapist's applications for these types of equipment. Objective data about the time a person spends using equipment over a week, month or longer, environments of use and performance in those environments, as well as rationales about the features and options that have been requested which will impact on wheelchair use including model of equipment, motor and battery capacity, seating and support options and type and location of wheelchair controller etc. are now required. There is a pressing need to augment the existing therapist's subjective assessments of PMD use with a more objective and quantitative performance indicators: "The use of subjective and objective assessments would provide complementary, but distinct, information allowing a more complete assessment of mobility" [16].

Yet it is important to emphasize that quantitative assessment does not mean a preference over subjective/qualitative assessment. Tthere is a need to complement, not to replace, one with the other. As rightly stated by one of the reviewers, therapists are highly skilled at combining observations with evaluation ("they see behaviour and can judge the quality of it simultaneously"). A therapist will typically choose an assessment tool, as outlined above, and then set up an assessment time and area in which to observe a person using a particular PMD. The assessment might last for several hours and is usually performed in the intended environment(s) of use. The observation might be repeated several times thereafter

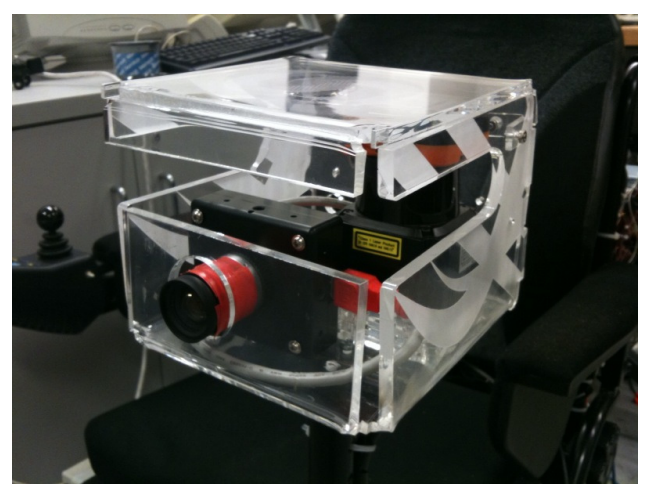

Fig. 2. Sensor package - detail.

to ensure that a thorough and un-biased evaluation can be completed. in fact, the environments in which a person uses a PMD should be assessed for up to 18 months after they have received the device [17]. If the therapist was able to be present at all times in which the person was using the chair an even more comprehensive evaluation could be completed. However, it is not possible for the therapist to be present for this length of time, nor is it possible to simulate all conditions in which the user will use the device - this includes the physical environments but also times of day, traffic and weather conditions, internal factors of the user, e.g. fatigue states etc. It is argued that the availability of additional objective information and logging capabilities will allow staff to make more conclusive analysis about the operation of PMDs by patients.

Work presented in this paper is based on a collaboration between the Centre for Autonomous Systems (CAS) at the University of Technology, Sydney (UTS) and the Prince of Wales (PoW) hospital with the aim of developing a standalone sensor package which is able to be deployed on a wheeled platform such as a wheelchair or a walker. The sensing platform includes a suite of sensors with a facility for data logging, whilst the algorithms developed determine the trajectory followed by the wheeled platform and generate a map of the surrounding environment. The proposed study describes a system which will allow therapists to obtain additional objective data about environments of use and operation of PMDs in those environments. The data will be interpreted by the team (including therapists) and used to support and augment the therapist's observations in completing a comprehensive evidence-based evaluation of a person's PMD use. This, in turn, will present occupational therapists with the capability to factually decide whether patients are ready to be safely deployed with mobile aids for their daily activities.

The paper is organised as follows: Section II provides a brief description of the sensor package, while an overview of the algorithms developed is given in Section III. Section IV presents the outcomes of the experiments carried out in the laboratory at UTS, and field tests conducted at the PoW Hospital in Sydney. Discussion, conclusions and future work are presented in Section V. 


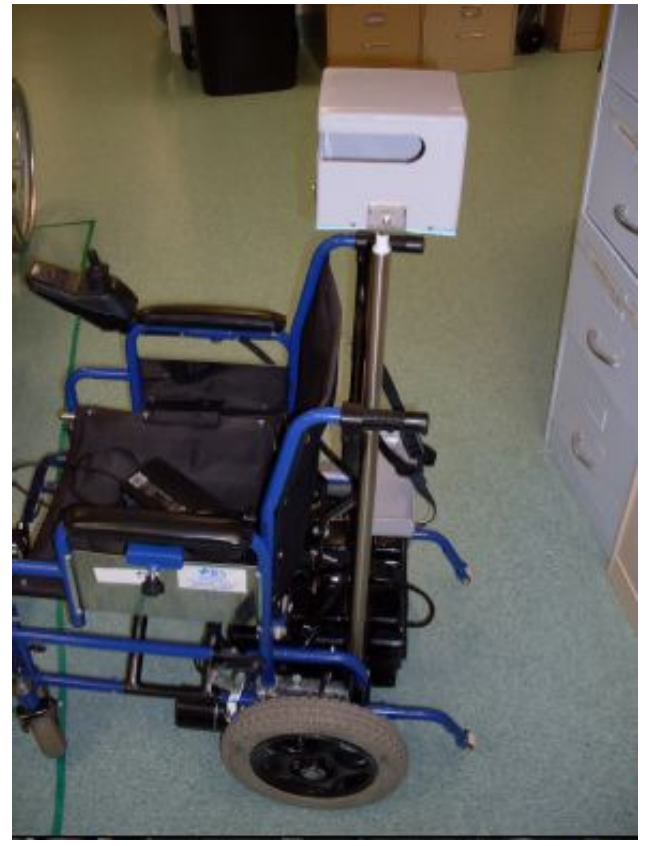

(a) Sensor enclosure mounted on wheelchair

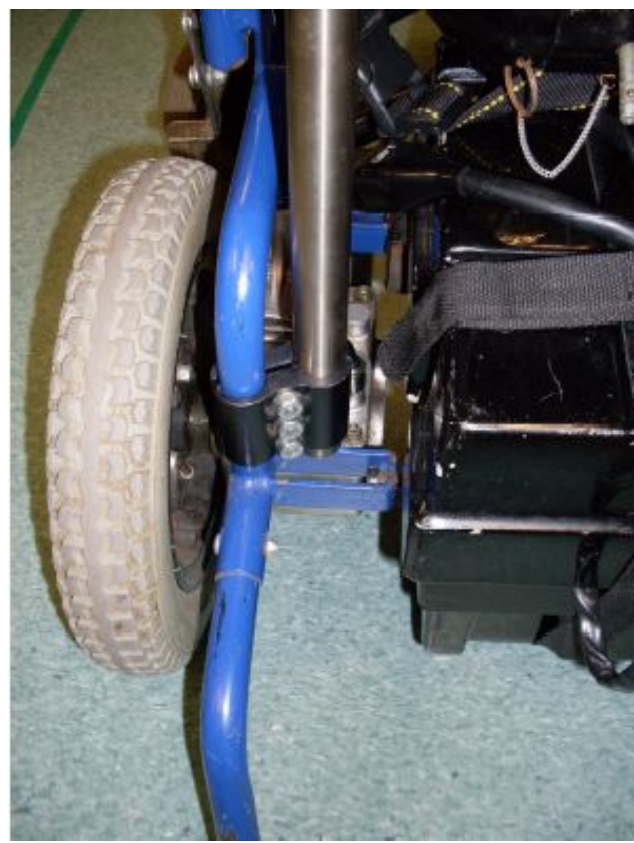

(b) Enclosure lower fixings

Fig. 3. Sensor enclosure mounted on a standard electric wheelchair at the PoW hospital.

\section{SENSOR PACKAGE}

The sensor package employed consists of:

- A Hokuyo UTM-30LX/LN scanning laser range finder, able to measure distance to objects between $\{0.1 \mathrm{~m}$ $30 \mathrm{~m}\}$ in a semicircular field of $270^{\circ}$.

- A Point Grey Dragonfly2 Firewire camera able to capture high resolution (1032x776pixels) colour images at 30fps.

- A Xsens MTi inertial measurement unit (IMU), a low weight 3DoF attitude and heading reference system capable of measuring accelerations, angular velocities and magnetic orientations.

A picture of the current sensor arrangement mounted on an automated wheelchair developed at UTS is shown in Figure 1, with a more detailed picture of the standalone enclosure depicting the configuration of the sensors being displayed in Figure 2. Based on this set-up, the actual enclosure designed to the requirements of the Biomedical Engineering department at the PoW hospital for deployment within the hospital grounds can be seen in Figure 3a.

The end product is a small size, light-weight, self-contained package that can be easily mounted on most mobility aids with standard fixtures, as shown in Figure $3 \mathrm{~b}$ for the case of a standard Pride LX electric wheelchair. This is one of the platforms regularly used by the occupational therapists at the PoW hospital in their routine assessments, and will also be used in the experiments presented in this paper. Further to the sensor set-up, a Toshiba Libretto U100 notebook computer and a custom Lithium-Polymer battery (and power converters) are hosted outside in a separate enclosure to power and operate the sensors, and provide the data logging capabilities. The approximate total weight for the sensor package enclosure is less than $1 \mathrm{Kg}$, i.e. safe to handle and easy to place in secured high-centered positions for a wider field of view, as is the case in the PoW hospital wheelchair.

The sensor package mounting on the UTS chair will block the driver's view, and is shown primarily to indicate that the sensor package is small and compact and can be mounted almost anywhere on the chair. This setup is mainly employed for development at UTS. However, the sensor package is typically located out of the driver's field of view as shown in the PoW wheelchair.

\section{MAPPING AND TRACKING Algorithms}

The software framework is based on ROS (www.ros.org), an advanced open source meta-operating system for robotic platforms. The "GMapping" package [18], which implements a simultaneous localization and mapping algorithm [19] based on a Rao-Blackwellized particle filter, has been employed to effectively learn occupancy grid maps from the 2D laser range data. The generated map can then be re-used to localize the platform in the learned environment producing the trajectories followed by the user as she/he drives the wheelchair around. For this approach to operate successfully both laser range data and wheelchair odometry need to be made available. However this can only be possible for customized platforms where wheel odometers have been fitted and interfaced with. A key feature of the proposed strategy is that of portability, so that the package can be easily fit to any mobility aid without further instrumentation. To that end, a novel estimation module has been developed which makes use of the IMU yaw rate to generate a reliable heading for the platform. Successive 


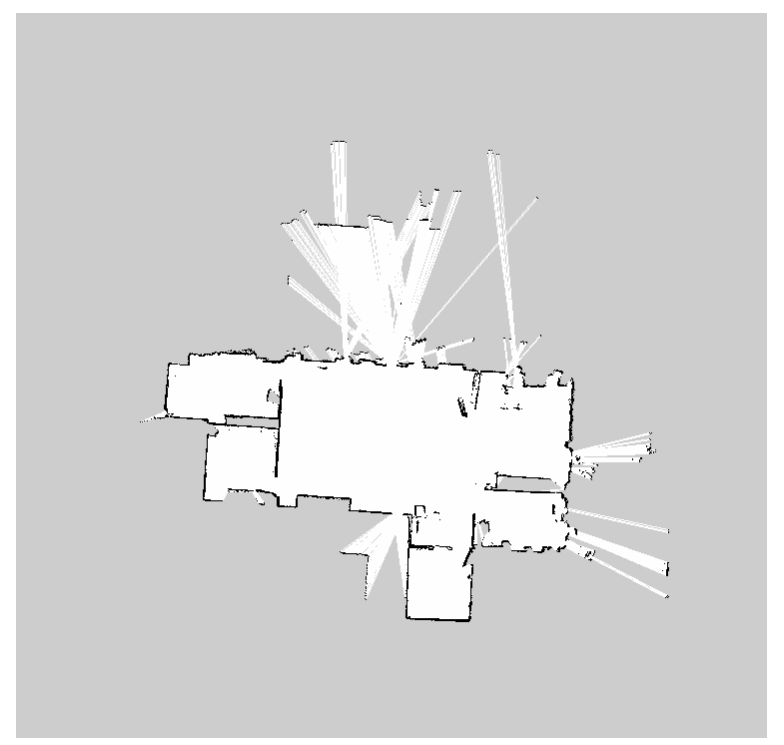

Fig. 4. Example of the resulting indoor map at the premises of the Occupational Therapy Department, PoW hospital. White is empty space, black is an obstacle (wall), grey is unknown (areas not seen by the sensors).

scans are then transformed by the orientation estimates and matched through an iterative closest point algorithm [20] to provide a measure of odometry information. This, in turn, can now be fed to either the GMapping module for initial lay-out composition, or a localization module such as the probabilistic Adaptive Monte-Carlo particle filter [21], built into ROS, for trajectory tracking.

It is important to note that maps need only be generated once for a given (static) environment. While generating these maps is a computationally expensive exercise, particularly for larger environments, localising the platform is a feasible proposition to be carried out on-line while driving around. For simplicity and validation, the trajectories hereby presented have been processed off-line.

\section{EXPERIMENTAL EVALUATIONS}

To assess the validity of the proposed hardware and software infrastructure in mapping and tracking the wheelchair platform in-situ, the sensor array was mounted on the standard PoW hospital wheelchair as illustrated in Figure 3. Data was then collected while the wheelchair was driven around the hospital environment by able users, both indoors and outdoors (please refer to the accompanying video for further details). Twodimensional (top-down view) maps of the environment like the one shown in Figure 4 were first built from the laser range finder and IMU data.

After the maps were created, the trajectory followed by the platform can be estimated. Figures $5 \mathrm{a}$ and $5 \mathrm{~b}$ depicts two such estimated trajectories. The former follows a relatively slow indoors-only trajectory, whilst the latter shows a combined indoors and outdoors run while the wheelchair was driven following more natural patterns of operation. From the logged data, it is now clearly possible for therapists and clinicians to examine the trajectory and other derived parameters in order to obtain a qualitative assessment of the user's driving ability to safely operate a PMD. There are a considerable number of quantitative registrations that can be measured using the sensor package, such as angular speeds, the closest distance between wheelchair and doorway when a user is asked to navigate through a door, or distance to a wall a user might be requested to follow in an exercise, can now be easily inferred. The choice of parameters is limited only by the requirements of the assessing team/therapist. Speed, for example, is an important observation and needs to vary depending on the environment of use. When crossing the road a wheelchair user will need to proceed quickly. However, when surrounded by other people or when approaching a doorway speed will need to be reduced. The sensor package will allow the assessing team/therapist to determine if a wheelchair user was able to select the speed appropriate to the environment of use and to accurately record and analyse this parameter as the person spends more time using the device. Similarly, if a wheelchair user's average speed was found to be low, as compared with that of a skilled user in a particular environment, it might be used to indicate the need to adjust parameters on the chair such as seating or position of the wheelchair controller. Alternatively, if the wheelchair was properly set up, such behaviour might suggest lack of confidence or the impact of any visual or cognitive impairment in a user and the need for further input by the therapist. For the purpose of illustration, the wheelchair linear velocity profile calculated for the path depicted in Figure $5 \mathrm{~b}$ is displayed in graphical form in Figure 6. The sensor package can acquire data for much longer periods than the $250 \mathrm{sec}$ period shown. The period of data acquisition would depend on the length of the assessment. The sensor package and associated algorithms effectively act as a "silent therapist or observer", naturally complementing the evaluations of clinicians and therapists.

\section{Discussion, CONCLUSIONS AND Future WORK}

Preliminary results from the current sensor package and the proposed estimation process are encouraging in being able to track the motion of a wheelchair user in the natural surroundings of a hospital. The availability of quantitative measures derived from the analysis of this data allows the therapist to augment current subjective assessments by more objective indicators.

During the prototyping phase of the project only data gathered from able bodied subjects was included in the development of the algorithms. Their comments have proved invaluable in developing the project to date. In future work, it is intended that data will be gathered using both skilled and unskilled users. The data will be used to establish norms for each group. The assessing team/therapist will be able to use these norms to determine how much training a new user will be required to become proficient in the use of the PMD. Alternatively, the data could be used to determine if there had been changes in the abilities of experienced users who might be challenged by progression of some condition or the occurrence of new pathology. 


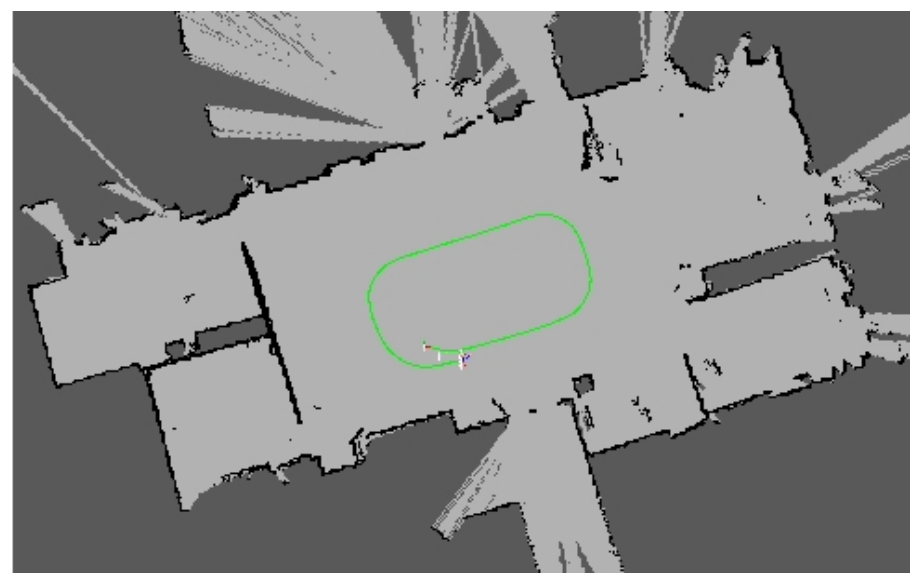

(a) Wheelchair trajectories at the PoW - indoors

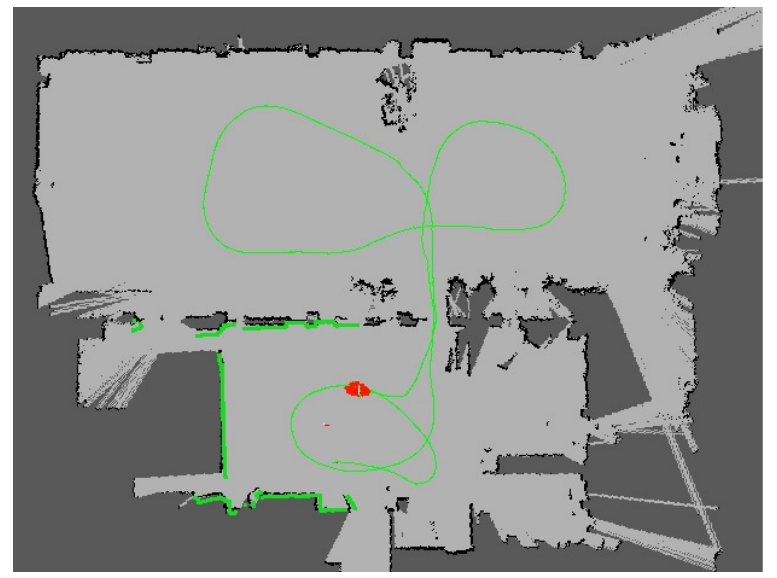

(b) Wheelchair trajectory at the PoW- indoors and outdoors

Fig. 5. Trajectories followed by the wheelchair platform during and indoor and outdoor test. Light grey is empty space, dark grey is unknown, green (B/W: grey in empty space) is the trajectory followed, white/red icon (B/W: white/dark gey) represents current wheelchair location.

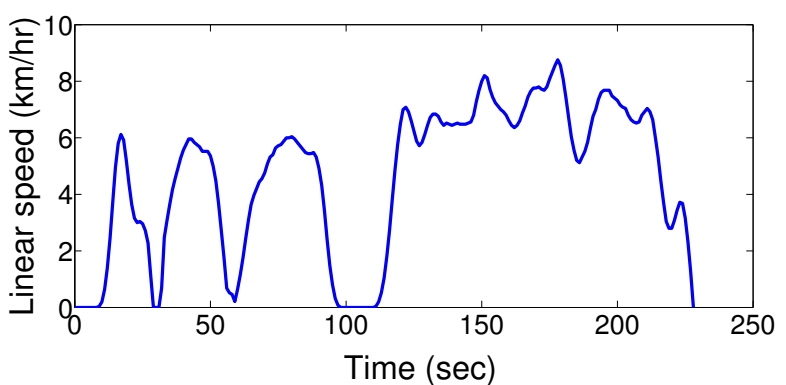

Fig. 6. Wheelchair linear speeds over the course of run depicted in Figure 5b.

In continuing with the current collaborative nature between robotic research engineers at UTS, and therapist and clinicians at the PoW hospital, future developments include studying the actual correlation (or augmentation) of the subjective information currently gathered by clinicians and therapists from the questionnaires used during assessment of PMDs, with the analytical data from the proposed sensor array and algorithms. Extensive tests in more realistic scenarios, and the development of software modules to enable the therapist to obtain information in an intuitive manner during the assessment process are currently under development or being planned as future work.

There are several phases to the proposed research. The first was to demonstrate that a sensor package can be mounted on a PMD, secondly that the device can produce objective data that enables therapists to augment their subjective observations. The last phase will propose the design of a device that could be made more autonomous and perhaps intervene where a therapist would have done so, e.g. slow the chair if a person approaches a wall or doorway too quickly or help guide a user through a complex environment. Our use of the term "silent therapist" is done quite deliberately. As one of the reviewers correctly noted, therapists are able to observe and evaluate simultaneously. Therapists can also intervene to ensure that a PMD user stays safe, or that a person completes a task by modelling or demonstrating "hand-over-hand" what is required, e.g. to navigate a particular path. The sensor package will initially allow the therapist to augment their observations. As therapists become familiar with the sensor package output they will be able to suggest algorithms which reflect their own thought processes, models and actions. These algorithms can then become embedded in the control system of the wheelchair and promote the safety and independence of the users and those around them. The sensor package/wheelchair control system will only ever be a support for, or reflection of, the decision making processes of the assessing team/therapist i.e. a "machine that thinks".

\section{ACKNOWLEDGMENT}

Part of this work was supported by the Australian Research Council $(A R C)$ through its Centre of Excellence programme, and by the New South Wales State Government. The ARC Centre of Excellence for Autonomous Systems $(C A S)$ is a partnership between the University of Technology Sydney, the University of Sydney and the University of New South Wales. The authors would like to thank Mr Peyman Biazar and Ms Janine Rudrum, from the Prince of Wales Hospital, for their assistance in the project.

\section{REFERENCES}

[1] S. Bricknell, Disability: the use of aids and the role of the environment. Disability Series, Australian Institute for Health and Welfare, Cat. No. DIS 32, 2003.

[2] Engineers Australia, "Policy statement - assistive technology and an ageing population," College of Biomedical Engineers, Policy Statement, 2010. [Online]. Available: http://www.engineersaustralia.org.au/da/index/getfile/id/9099

[3] P. Clarke and A. Colantonio, "Wheelchair use among community dwelling older adults: prevalence and risk factors in a national sample," Canadian Journal on Aging, vol. 24, pp. 191-198, 2005.

[4] H. S. Kaye, T. Kang, and M. P. LaPlante, Mobility Device Use in the United States. Disability Statistics Report 14. Disability Statistics Center, University of California, San Francisco, 2000. 
[5] K. Huhn, P. Guarrera-Bowlby, and J. E. Deutsch, "The clinical decisionmaking process of prescribing power mobility for a child with cerebral palsy," Pediatric physical therapy : the official publication, vol. 19, pp. 254-260, 2007.

[6] P. Axelson, J. Minkel, A. Perr, and D. Yamada, The Powered Wheelchair Training Guide. PAX Press, Minden, Nevada, USA 2002, 2002.

[7] F. Harris and S. Springle, "Outcomes measurement of a wheelchair intervention," Disability Rehabilitation Assistive Technology, vol. 3, pp. 171-180, 2008.

[8] A. D. Mountain, R. L. Kirby, and C. Smith, "The wheelchair skills test, version 2.4: Validity of an algorithm-based questionnaire version," Archives of Physical Medicine and Rehabilitation, vol. 85, pp. 416-426, 2004.

[9] T. Mills, M. B. Holm, E. Trefler, M. Schmeler, S. Fitzgerald, and M. Boninger, "Development and consumer validation of the functional evaluation in a wheelchair (few) instrument," Disability Rehabilitation Assistive Technology, vol. 24, pp. 38-46, 2002.

[10] D. Dawson, R. Chan, and E. Kaiserman, "Development of the powermobility indoor driving assessment for residents of long term care facilities," Canadian Journal on Aging, vol. 61, pp. 269-276, 1994.

[11] L. Letts, D. Dawson, I. Bretholz, E. Kaiserman-Goldenstein, J. Gleason, E. McLellan, L. Norton, and C. Roth, "Reliability and validity of the power-mobility community driving assessment," Assistive Techonology, vol. 19, pp. 154-163, 2007.

[12] T. Bernd, D. V. D. Pijl, and L. P. D. Witte, "Existing models and instruments for the selection of assistive technology in rehabilitation practice," Scandinavian Journal of Occupational Therapy, vol. 16, pp. $146-158,2009$.

[13] A. Souza, A. Kelleher, R. Cooper, R. A. Cooper, L. I. Iezzoni, and D. M. Collins, "Multiple sclerosis and mobility-related assistive technology: systematic review of literature," Journal of Rehabilitation Research and Development, vol. 47, no. 3, pp. 213-223, 2010.

[14] O. Fliess-Douer, Y. C. Vanlandewijck, G. Lubel Manor, and L. H. V. Van Der Woude, "A systematic review of wheelchair skills tests for manual wheelchair users with a spinal cord injury: towards a standardized outcome measure," Clinical Rehabilitation, vol. 24, no. 10, pp. 867-886, 2010. [Online]. Available: http://cre.sagepub.com/content/24/10/867.abstract

[15] W. B. Mortenson, W. C. Miller, J. Boily, B. Steele, E. M. Crawford, and D. G, "Overarching principles and salient findings for inclusion in guidelines for power mobility use within residential care facilities," Journal of Rehabilitation Research and Development, vol. 43, no. 2, pp. 199-208, 2006

[16] S. K. Wilson, J. P. Hasler, P. M. Dall, and G. M. H, "Objective assessment of mobility of the spinal cord injured in a free-living environment," Spinal Cord, vol. 46, no. 5, pp. 352-357, 2008.

[17] C. Auger, L. Demers, I. Glinas, W. C. Miller, J. W. Jutai, and L. Noreau, "Life-space mobility of middle-aged and older adults at various stages of usage of power mobility devices," Archives of Physical Medicine and Rehabilitation, vol. 91, no. 5, pp. 765-773, 2010.

[18] G. Grisetti, C. Stachniss, and W. Burgard, "Improving grid-based slam with rao-blackwellized particle filters by adaptive proposals and selective resampling," Proceedings of the IEEE International Conference on Robotics and Automation, pp. 2432-2437, 2005.

[19] W. Zhou, J. V. Miro, and G. Dissanayake, "Information-efficient 3D visual SLAM for unstructured domains," IEEE Transactions on Robotics, vol. 24, no. 5, pp. 1078-1087, 2008.

[20] P. J. Besl and H. D. McKay, "A method for registration of 3-d shapes," IEEE Transactions on Pattern Analysis and Machine Intelligence, vol. 14, no. 2, pp. 239-256, 1992.

[21] D. Fox, W. Burgard, F. Dellaert, and S. Thrun, "Monte carlo localization: Efficient position estimation for mobile robots," in Proceedings of the Sixteenth National Conference on Artificial Intelligence (AAAI'99), 1999, pp. 343-349. 